\title{
Análise de capital social e qualidade de vida da população rural: Um estudo de caso no munícipio de Itarema, estado do Ceará
}

\author{
Francisco José Silva Tabosa ${ }^{1}$ \\ Ruben Dario Mayorga ${ }^{2}$ \\ Jair Do Amaral Filho ${ }^{3}$ \\ Ahmad Saeed Khan ${ }^{4}$
}

\begin{abstract}
Resumo: O presente estudo tem como objetivo determinar e analisar as disparidades de desenvolvimento existentes nas comunidades de Porto dos Barcos e Oriente no município de Itarema. Para isso, elaboraram-se dois tipos de questionário: um na tentativa de encontrar as características tangíveis do capital social existente nas duas comunidades e outro, na tentativa de mensurar a qualidade de vida existente nas comunidades. Os resultados mostraram que a Comunidade de Oriente, escolhida pelo "Conselho de Conhecedores" como a mais desenvolvida, possui um maior estoque de capital social quando comparada com a Comunidade de Porto dos Barcos, desfrutando de uma melhor qualidade de vida.
\end{abstract}

Palavras-chave: capital social; qualidade de vida; município de Itarema.

${ }^{1}$ Doutorando em Economia no CAEN/UFC. E-mail: franze@.caen.ufc.br

${ }^{2}$ Prof Associado do Departamento de Economia Agrícola da Universidade Federal do Ceará. E-mail: Dario@ ufc.br

${ }^{3}$ Prof. Titular do Departamento de Teoria Econômica e do Curso de Pós-Graduação em Economia - CAEN - da Universidade Federal do Ceará. E-mail: amarelo@fortalnet.com.br

${ }^{4}$ Prof. Titular do Departamento de Economia Agrícola da Universidade Federal do Ceará. Pesquisador do CNPq. E-mail: saeed@ufc.br 


\title{
Analysis of the social capital and quality of life of the rural population in Itarema county, Ceará state
}

\begin{abstract}
The present study has as objective to determine and to analyze the development disparities among Porto dos "Barcos" and "Oriente" in Itarema County, resulting from the presence/absence of social capital. For this, one prepared two kinds of questionnaires: the first one, in the attempt of showing the real characteristics of social capital existent in two communities, and other one, in the attempt of measuring the quality of life in the communities, looking for create an index of social capital as quality of life. The results show that the community of "Oriente" chosen as the most develops for the "Conselho de Conhecedores", has bigger stock of social capital when compared with Porto dos Barcos community, the least developed, enjoy a better quality of life.
\end{abstract}

Key words: social capital; quality of life; Itarema county.

JEL: R51, R11

\section{Introdução}

A partir da década de 80 surge uma indagação, nos estudos sobre o desenvolvimento econômico. Esses estudos buscavam saber a razão pela quais algumas regiões cresciam e se desenvolviam e outras não e por que umas desenvolvem mais do que outras; mesmo que estas regiões disponham de condições similares em termos de fatores produtivos - capital físico, tecnologia e mão de obra (Amaral Filho 2000).

Existem quatro formas básicas de capital, que podem ser utilizadas para analisar o desenvolvimento de uma região, conforme o WORLD BANK (2004): capital natural (recursos naturais); capital físico (tecnologia, equipamentos e o sistema financeiro); capital humano (níveis existentes de educação e saúde); e capital social (capital referente às instituições, relações sociais e normas). Essa última forma de capital conforma o aspecto da qualidade e a quantidade das interações sociais de uma sociedade, tornando-se objeto de resposta para o sucesso ou fracasso do desenvolvimento de algumas regiões.

Existem várias formas de definir capital social. Putnam (1996: 177) define capital social como “(...) características de organização social, como confiança, normas e sistemas, que contribuem para aumentar a eficiência da sociedade, facilitando as ações coordenadas”. Em outras palavras, constituindo-se, o capital social, em um fator determinante para o desenvolvimento econômico e social, através de uma maior participação, organização e cooperação horizontal 
entre os atores sociais, com objetivo de fortalecer o tecido social.

O capital social surge como uma resposta da Teoria Endogeneista Institucionalista ${ }^{5}$ indagação aos estudos de desenvolvimento econômico, em relação às disparidades de crescimento de determinadas regiões. Em torno da ideia de que é insuficiente considerar apenas os aspectos materiais e tangíveis da vida econômica. Levam agora em consideração alguns aspectos da sociedade e as suas relações sociais; sendo necessária à consideração de novos fatores, de caráter decisivo na produção, na comercialização e na articulação políticoinstitucional, determinados na própria região de forma endógena, passando a serem vistos de forma diferente. Onde a região que consegue reunir esses fatores possui melhores condições de atingir um desenvolvimento mais equilibrado.

Para Amaral Filho (2000) e Mayorga et al. (2004) comunidades com maiores níveis de capital social são mais propensas a se desenvolverem do que comunidades com baixos níveis de capital social. Isso se deve ao benefício que surge do acúmulo de articulações sociais e ao grau de organização da sociedade, gerando melhorias na qualidade de vida da população e criando alternativas para superar os problemas existentes na região (Kliksberg 1999).

Nesse contexto, verifica-se que o município de Itarema com uma população de 30.347 habitantes em 2000 conseguiu melhorar o seu Índice de Desenvolvimento Municipal (IDM), entre os anos de 1997 e 2002, passando de 19,31 para 22,70; representando uma melhora relativa de 17,55\%. Melhora equivalente ao nível de municípios como Camocim, Marco e Acaraú (município do qual Itarema foi distrito até 1985), e superior à de todos os outros municípios que compõem a Microrregião do Litoral de Camocim e Acaraú. Observa-se também que na escala em nível estadual, no que diz respeito ao IDM, Itarema passou de $139^{\text {a }}$ colocado para a $113^{\text {a }}$ posição (IPLANCE 2001; IPECE 2004).

No entanto, esse município vivencia disparidades de desenvolvimento entre suas comunidades devido, em parte, a existência desigual de capital social comunitário, determinante de diversos níveis de qualidade de vida.

Assim sendo, considera-se a existência e acúmulo de capital social como um importante agente de transformação, modernização e articulação das comunidades do município de Itarema. Ou seja, espera-se que o impacto da existência ou carência de capital social seja refletido nos níveis de qualidade de vida das comunidades no município de Itarema.

\footnotetext{
${ }^{5}$ A Teoria Endogeneista possui duas correntes: a Teoria do Crescimento Endógeno, que consiste na ruptura da Teoria Tradicional do Crescimento (representado pelo modelo de SOLOW), cujos fundadores parecem terem sido os novos clássicos (LUCAS e ROMER na década de 80) e a Corrente Institucionalista, mais ligada ao desenvolvimento local e regional e ao capital social, que aborda “... o processo de ampliação contínua da capacidade de absorção interna da região, cujo desdobramento é a retenção do excedente econômico gerado na economia local e/ou a atração de excedentes provenientes de outras regiões. Isso resulta na ampliação do emprego, do produto e da renda do local ou da região, dentro de um modelo de desenvolvimento regional definido" (Amaral Filho 1995).
} 
O objetivo geral deste estudo é determinar e analisar as disparidades de desenvolvimento existentes nas comunidades de Porto dos Barcos e Oriente do município de Itarema, decorrentes da presença/ausência de capital social.

Especificamente, o estudo busca: a) analisar o acúmulo de capital social nas comunidades de Porto dos Barcos e Oriente; $b$ ) verificar os fatores determinantes da qualidade de vida das famílias nas comunidades de Porto dos Barcos e Oriente e sua relação com o estoque de capital social existente; c) identificar o impacto da articulação político-institucional entre as comunidades estudadas e a prefeitura de Itarema.

O presente trabalho será dividido em seções. Inicialmente, apresenta-se a introdução com a definição do problema. Na seção 1 será apresentado o referencial teórico. A seguir será abordado o procedimento metodológico, logo os resultados e discussão e, finalmente, as conclusões do estudo.

\section{Referencial Teórico}

\subsection{Capital Social}

O capital social é um termo que foi utilizado primeiramente na Sociologia, em trabalhos produzidos por Pierre Bourdieu e James Coleman (considerado por muitos pesquisadores, um dos responsáveis pela utilização do capital social nas agendas de pesquisa) na década de 8o. Posteriormente, o capital social surge na Ciência Econômica através, principalmente, do trabalho realizado por Robert Putnam, na sua obra "Comunidade e Democracia: a experiência da Itália Moderna” de 1996.

Existem três aspectos que diferenciam o capital social das outras formas de capital. O primeiro está na sua intangibilidade: conforme Amaral Filho (2000), apesar da dificuldade de se medir, é possível afirmar que o capital social esteve por trás dos sucessos de desenvolvimento de muitas regiões, e a sua ausência esteve por trás de muitos fracassos. O segundo é o fato de ele normalmente constituir um bem público, ao contrário do capital convencional, que normalmente é um bem privado (Putnam 1996). O terceiro é que ele não se deprecia com o tempo, de acordo com Ostrom (1990), algumas das características do capital social são mais perceptíveis quando este é comparado com o capital físico: quanto maior a sua utilização, maior será o estoque de capital social, "ao contrário das máquinas que sofrem de depreciação, a utilização freqüente de uma norma ou de um vínculo de confiança só contribui para o seu fortalecimento e a sua disseminação. A degeneração do capital social vem da sua falta de uso" (Monastério 1999:3).

Conforme Putnam (1996: 174), só existe capital social com a existência de confiança mútua entre os indivíduos (reciprocidade): Para haver cooperação é preciso não só confiar nos outros, mas também acreditar que goza da con- 
fiança dos outros. Sendo assim, o referido autor vê na confiança a resolução dos dilemas da ação coletiva, na qual os indivíduos, mesmo interessados em agir cooperativamente, não conseguem gerar resultados coletivamente eficientes. Já para Ostrom (1990), a organização social constitui o capital social, facilitando a realização dos objetivos que não poderiam ser conseguidos em sua ausência, mas que poderiam ser conseguidos somente, a um custo mais elevado.

Balland e Platteau (1999; 2003) comentam que as desigualdades de desenvolvimento local podem ser explicadas pela interação e ação coletiva entre os atores sociais. Regiões onde a desigualdade é maior, os pobres passam a ter menos potencial de riqueza, enfrentando assim barreiras de ação coletiva. Para os autores, o capital social proporciona uma valorização das ações coletivas, buscando sempre reduções das desigualdades entre regiões.

Segundo o cientista social John Durston (1999:103) “o termo capital social faz referencia às normas, instituições e organizações que promovem a confiança e a cooperação entre as pessoas, as comunidades e no conjunto da sociedade".

$\mathrm{O}$ referido autor em seus trabalhos em comunidades rurais na Guatemala define uma nova forma de capital social: o capital social comunitário. " $E l$ capital social comunitario es una forma particular de capital social, que abarca el contenido informal de las instituciones que tienen como finalidad contribuir al bien común." (Durston 1999: 103 - 104).

Abrahan e Platteau (2001; 2004) afirmam que tanto as comunidades rurais africanas, como na América Latina no século passado, foram conduzidas frequentemente por líderes ditatoriais que dominavam o processo de participação coletiva, impossibilitando a cooperação intra e entre grupos, e portanto a mudança social, resultando em baixos níveis de desenvolvimento dessas regiões.

Abu-El-Haj (2000), Holanda (2003), Tabosa et al. (2004), Mayorga et al.(2004), Khan e Silva (2005), Barreto e Khan (2006) e Tabosa e Mayorga (2006) analisaram o capital social em comunidades rurais no Estado do Ceará e concluíram que comunidades, com fortes aspectos de liderança, confiança mutua, cooperação e articulação social se destacavam em relação às demais, devido à eficiência institucional no atendimento a suas reivindicações. Segundo os autores, o fortalecimento da capacidade de ação dos pobres para melhorar a situação na qual se encontravam, a cooperação foi fundamental para o desenvolvimento dessas comunidades. 


\subsection{Qualidade de Vida}

Durante muito tempo, utilizou-se a renda per capita como um meio de medir bem-estar de uma população. Mas este instrumento, sozinho, revela-se insuficiente para avaliar a qualidade de vida de uma população. A partir de 1960, a Organização das Nações Unidas (ONU) passou a utilizar o Índice de Desenvolvimento Humano (IDH), para avaliar as condições de vida dos 174 países membros da organização. Criou-se um ranking em três áreas: educação, renda e expectativa de vida.

Conforme Nilander e Mathis (2001) desde a década de 90 tem-se desenvolvido várias experiências no sentido de construir indicadores, e índices de condições de vida da população como instrumentos de planejamento e formulação de políticas públicas.

Segundo Gómez (2000), citado por Meregue et al. (2000), “definir qualidade de vida implica em formas inéditas de identidade, cooperação, solidariedade, participação e realização, assim como satisfação de necessidades e aspirações tendo em vista as transformações sociais” (Gómez 2000: 2).

Já Khan (2002), apesar de não existir um conceito claro do que é qualidade de vida, "a definição do nível de vida deve ser entendida como um estado atual de suas condições concretas de vida e não como um estado desejado" (Khan 2002: 273).

Ainda em relação ao tema, Khan (2000) comenta que o tamanho do PIB per capita de uma população, utilizado anteriormente como único indicador para medir o bem-estar, não é suficiente para analisar as condições de vida. Faz-se necessário, segundo o autor, buscar medidas socioeconômicas mais abrangentes, como bens duráveis, saúde, educação, habitação e aspectos sanitários.

\section{Procedimento Metodológico}

\section{1 Área de Estudo}

A área de estudo compreende o município de Itarema (palavra originária do tupi que significa pedra de cheiro desagradável). Localizado a $220 \mathrm{Km}$ de Fortaleza, na microrregião do Litoral de Camocim e Acaraú (IPLANCE 2001; IPECE 2004). 


\subsubsection{Justificativa da área de estudo}

As Comunidades de Porto dos Barcos e Oriente estão localizadas no município de Itarema e foram selecionados por apresentarem níveis diferentes de desenvolvimento, segundo o Conselho de Conhecedores ${ }^{6}$; além de dados obtidos junto às secretarias municipais de ação social, agricultura, saúde e educação.

A Comunidade de Porto dos Barcos está formada por 100 famílias e localizada no litoral do município de Itarema, a $6 \mathrm{~km}$ da sede municipal. A sua principal atividade econômica é a pesca, principalmente de lagosta. Outra atividade econômica em desenvolvimento na comunidade é o artesanato, com a produção de redes de pesca, bordados, crochês e bijuterias; predominando o trabalho das mulheres dos pescadores.

A Comunidade de Oriente está formada por 79 famílias e localizada no sertão de Itarema, no distrito de Carvoeiro, a $32 \mathrm{~km}$ da sede municipal. A atividade econômica predominante é a agropecuária, destacando a produção de feijão, arroz e mandioca e, no verão, a produção do caju (venda da castanha de caju para um comerciante do próprio município, que repassa para a CIONE - uma das maiores empresas de castanha de caju do Estado do Ceará). Também, vale ressaltar, a criação de bovinos e aves.

\subsection{Fontes de Dados}

Os dados primários utilizados na análise provêm da aplicação de questionários a membros específicos da comunidade que formam o que denominaremos nesta pesquisa "Conselho de Conhecedores" do município de Itarema. As famílias, para a análise da qualidade de vida, foram escolhidas aleatoriamente das associações comunitárias das comunidades estudadas ${ }^{7}$, totalizando 40 famílias; com a finalidade de construir os Índices de Capital Social e Qualidade de Vida, respectivamente. Os próprios autores aplicaram os questionários.

Os dados secundários utilizados provêm do IPLANCE (Instituto de Planejamento do Estado do Ceará), do IPECE (Instituto de Pesquisa e Estratégia Econômica do Ceará); além de uma ampla revisão de literatura sobre os temas de capital social e qualidade de vida; desde livros, dissertações, pesquisas até publicações avulsas, jornais, revistas e textos obtidos via Internet.

${ }^{6} \mathrm{O}$ Conselho de Conhecedores foi formado por pessoas de diversos órgãos como outros membros da sociedade civil de Itarema, conhecedores deste município. Mais detalhes na seção 2.3.

${ }^{7}$ No caso da Comunidade de Porto dos Barcos, as associações foram: Associação Comunitária dos Moradores do Bairro Alegre e a Associação de Artesanato de Porto dos Barcos. Já em relação à Comunidade de Oriente foi a Associação Comunitária dos Moradores de Oriente. 


\subsection{Métodos de Análise e Técnicas de Pesquisa}

Foram utilizados os métodos: observacional, comparativo e monográfico. As técnicas de pesquisa utilizadas foram: pesquisa de campo, pesquisa bibliográfica, pesquisa documental, levantamentos e estudo de caso.

No primeiro momento, foi realizado um contato junto às lideranças e representantes do município de Itarema (prefeito, secretários, padres, líderes comunitários, presidentes de associações, cooperativas e sindicatos, EMATERCE, etc), enfim, pessoas que realmente conheçam todo o município, com o objetivo identificar a comunidade mais desenvolvida e mais pobre do município.

No segundo momento, após definir as duas comunidades a serem estudadas, criou-se o "Conselho de Conhecedores", formado por líderes das duas comunidades estudadas, representantes das secretarias de ação social, saúde e agricultura de Itarema, diretoria do Sindicato dos Trabalhadores Rurais de Itarema, que conheciam bem as duas comunidades escolhidas.

Em seguida, foram aplicados dois tipos de questionário: um para os integrantes do "Conselho de Conhecedores" baseado no questionário utilizado pelo Banco Mundial (Grootaert et al. 2003) para medir o capital social existente nas duas comunidades, e outro, para as famílias selecionadas, sobre qualidade de vida nas comunidades para a construção do índice proposto.

O questionário do Índice de Capital Social aborda questões relevantes sobre os indicadores determinantes de capital social, de acordo com o Questionário Integrado para Medir Capital Social do Banco Mundial ${ }^{8}$ : grupos e redes, confiança e solidariedade, ação coletiva e cooperação, informação e comunicação, coesão e inclusão social e autoridade ou capacitação e ação política.

- Grupos e Redes: indicador que considera a natureza e a extensão da participação de vários tipos de organizações sociais e redes informais nas comunidades. Considera, também, os aspectos de liderança e o envolvimento da comunidade com os grupos. Espera-se que uma comunidade que tenha maior quantidade de grupos ou associações e lideranças participativas, maior será a sua capacidade de gerar e acumular capital social;

- Confiança e Solidariedade: são duas das principais características de capital social. Esse indicador busca levantar dados sobre a confiança e solidariedade existentes na comunidade. Espera-se que, quanto maiores os índices de confiança e solidariedade existentes entre os membros de uma comunidade, maior será o capital social existente naquela comunidade;

- Ação Coletiva e Cooperação: esse indicador investiga se e como os membros de uma comunidade têm trabalhado com outras pessoas em sua comunidade em projetos comuns e se existem consequências pela participação nessas atividades. Espera-se que uma comunidade em que seus membros

${ }^{8}$ Ver Grootaert et al. (2003). 
detenham espírito de coletividade e cooperação, possua maior estoque de capital social;

- Informação e Comunicação: indicador que explora os meios pelos quais a comunidade recebe informações relativas às condições de mercado e serviços públicos. Espera-se que uma comunidade que receba maior número de informações relevantes sobre as condições de mercado e serviços públicos terá maior capacidade de gerar e acumular capital social;

- Coesão e Inclusão Social: indicador que explora várias formas de divisão e diferenças que podem levar ao conflito dentro de uma comunidade, dentre elas a exclusão de serviços públicos essenciais, a violência e exclusão social. Espera-se que comunidades com baixos níveis de desigualdades, violência e exclusão social entre seus moradores tenham maior capacidade de gerar e acumular capital social;

- Autoridade ou Capacitação e Ação Política: indicador que mede se a comunidade detém certo controle sobre instituições e processos que afetam diretamente o seu bem-estar. Espera-se que comunidades com maior mobilização popular detenham maior estoque de capital social.

As ponderações de cada indicador foram atribuídas, tanto pelo Conselho de Conhecedores quanto por especialistas sobre capital social no Estado do Ceará, de acordo com sua importância relativa.

Já o questionário do Índice de Qualidade de Vida aborda questões relevantes sobre os indicadores determinantes de qualidade de vida: informação e comunicação, conflito e violência, saúde e educação, habitação, condições sanitárias e indicador econômico.

A metodologia para a construção do Índice de Qualidade de Vida (IQVi) de uma comunidade, seguiu a sugerida por Khan (2000), com os seguintes procedimentos: estabelecimento dos indicadores que compõem o índice de qualidade de vida e atribuição dos escores as variáveis que integraram cada indicador. Neste índice foram atribuídos pesos iguais para todos os indicadores que compõem este índice.

1. Indicador Informação e Comunicação: indicador que verifica a distância da comunidade em relação à sede do município, os acessos à estrada, ao serviço de telefonia, rádio e televisão.

2. Indicador Conflito e Violência: indicador que verifica o grau de violência existente na comunidade.

3. Indicador Saúde e Educação: indicador que verifica a disponibilidade de serviços de saúde e educação na comunidade.

4. Indicador Habitação: indicador que verifica o tipo de construção e iluminação das residências de uma comunidade. Espera-se que a comunidade 
com melhores residências, em relação à construção e iluminação, terá melhor qualidade de vida.

5. Indicador Condições Sanitárias e Higiene: indicador que verifica o destino dado aos dejetos humanos, ao tratamento da água para consumo humano e o lixo domiciliar. Espera-se que quanto melhores forem às condições sanitárias de uma comunidade melhor será a qualidade de vida.

6. Indicador Econômico: indicador que verifica a renda média mensal e os bens duráveis das famílias de uma comunidade. Espera-se que a comunidade com melhor renda e mais e melhores bens duráveis terá melhor qualidade de vida.

\section{Resultados e Discussão}

Nesta seção serão apresentados os resultados sobre o Índice de Capital Social e o Índice de Qualidade de Vida nas Comunidades de Porto dos Barcos e de Oriente do município de Itarema, Ceará.

\section{1 Índice de Capital Social nas Comunidades de Porto dos Barcos e Oriente}

$\mathrm{Na}$ Tabela 1 constam os valores relacionados ao Índice de Capital Social nas Comunidades de Oriente e de Porto dos Barcos do município de Itarema.

TABELA 1 - PONDERAÇÃO DOS INDICADORES, ESCALA DE DESEMPENHO E ÍNDICE DE CAPITAL SOCIAL NAS COMUNIDADES DE ORIENTE E DE PORTO DOS BARCOS, 2005

\begin{tabular}{|c|c|c|c|c|c|c|}
\hline Indicadores $\left(\mathbf{X}_{\mathrm{i}}\right)$ & $\mathbf{P i}^{1}$ & $\mathbf{E D}_{1 i}{ }^{2}$ & $\mathbf{E D}_{2 \mathrm{i}}{ }^{3}$ & $\mathrm{ICS}_{1 \mathrm{i}}{ }^{4}$ & $\mathrm{ICS}_{2 \mathrm{i}}{ }^{5}$ & RED $_{i}{ }^{6}$ \\
\hline Grupos e Redes $\left(\mathrm{X}_{1}\right)$ & 0,20 & 0,56 & 0,78 & 0,11 & 0,16 & 1,39 \\
\hline Confiança e Solidariedade $\left(\mathrm{X}_{2}\right)$ & 0,23 & 0,69 & 0,99 & 016 & 0,23 & 1,43 \\
\hline Ação Coletiva e Cooperação $\left(X_{3}\right)$ & 0,21 & 0,62 & 0,77 & 0,13 & 0,16 & 1,25 \\
\hline Informação e Comunicação $\left(\mathrm{X}_{4}\right)$ & 0,11 & 0,83 & 0,71 & 0,09 & 0,08 & 0,85 \\
\hline Coesão e Inclusão Social $\left(\mathrm{X}_{5}\right)$ & 0,13 & 0,71 & 0,89 & 0,09 & 0,12 & 1,25 \\
\hline $\begin{array}{l}\text { Autoridade ou Capacitação e Ação } \\
\text { Política }\left(\mathrm{X}_{6}\right)\end{array}$ & 0,12 & 0,70 & 0,83 & 0,08 & 0,10 & 1,19 \\
\hline Total & 1,00 & & & 0,66 & $\mathbf{0 , 8 5}$ & $1,29^{7}$ \\
\hline
\end{tabular}

Fonte: Dados da pesquisa

1 - Ponderação dos Indicadores

2 - Escala de Desempenho na Comunidade de Porto dos Barcos

3 - Escala de Desempenho na Comunidade de Oriente

4 - Índice de Capital Social na Comunidade de Porto dos Barcos $\left(\operatorname{ICS}_{1 \mathrm{i}}=\sum \mathrm{Pi}^{*} \mathrm{ED}_{1 \mathrm{i}}\right)$

5 - Índice de Capital Social na Comunidade de Oriente $\left(\operatorname{ICS}_{2 \mathrm{i}}=\sum \mathrm{P}_{\mathrm{i}}^{*} \operatorname{ED}_{2 \mathrm{i}}\right)$

6 - Relação entre as Escalas de Desempenho $\left(\operatorname{RED}_{\mathrm{i}}=\mathrm{ED}_{2} / \mathrm{ED}_{1}\right)$

7 - Relação entre o Índice de Capital Social $\left(\mathrm{RICS}=\mathrm{ICS}_{2} / \mathrm{ICS}_{1}\right)$ 
Os indicadores de capital social foram ponderados segundo a sua importância para as comunidades, atribuída pelo "Conselho de Conhecedores" de Itarema e especialistas sobre capital social no Estado do Ceará, apresentados na COLUNA 2.

Os valores da COLUNA 3 são as Escalas de Desempenho de cada indicador determinante do capital social na Comunidade de Porto dos Barcos.

A Comunidade de Porto dos Barcos obteve melhor desempenho no indicador Informação e Comunicação (ED14 = 0,83), onde o desempenho chegou a 83\%. Isso significa que a Comunidade de Porto dos Barcos tem forte acesso às informações relevantes ao mercado e serviços públicos, principalmente porque a comunidade fica distante apenas $6 \mathrm{~km}$ da sede do município, com estrada asfaltada e acessível durante o ano todo.

Apesar do indicador Coesão e Inclusão Social (X5) ter apresentado um desempenho de 71\%, a Comunidade de Porto dos Barcos vivencia problemas de exclusão social, com desigualdades sociais. Principalmente entre um pequeno número de donos de embarcações e um grande número de pescadores, que ganham por Kg de peixe pescado. Apresentaram-se na comunidade elevados casos de alcoolismo e de violência familiar' ${ }^{9}$

No indicador Grupos e Redes ( $\mathrm{X}_{1}$ ), o desempenho da Comunidade de Porto dos Barcos foi de 56\%, devido às associações existentes não serem articuladas e não terem forte representação em reuniões e reivindicações junto ao governo local.

As associações mais importantes da comunidade são: Associação Comunitária dos Moradores do Bairro Alegre e a Associação de Artesanato de Porto dos Barcos, que possuem muitos associados. No entanto, poucos participam de atividades comunitárias (reuniões mensais, festas beneficentes, petições, mutirão, etc). Só participam quando solicitados a receberem algum benefício, como hora de plantar, bolsa família, seguro para os lagosteiros; ou algum caso de problema, como abastecimento de água, doença ou morte.

As duas lideranças da comunidade, Maria Joraci da Silva (Dona Zeza), presidenta da Associação de Artesanato de Porto dos Barcos e Maria de Fátima Santos Silva, presidenta da Associação Comunitária dos Moradores do Bairro Alegre são as duas únicas pessoas que frequentam reuniões na prefeitura local e no sindicato dos trabalhadores. No entanto, são pessoas pouco participativas 
e não são estimuladas pelos próprios membros das associações a participarem com mais frequência.

Segundo Dona Zeza, a maioria dos moradores de Porto dos Barcos vivem suas vidas isoladamente. Poucos são os que se preocupam com a vida do vizinho ou de um amigo. Ela acha difícil existir confiança entre os moradores, pois cada um quer levar vantagem sobre o outro, principalmente quando se trata de benefícios do governo, como o bolsa família, dentre outros.

Em relação ao indicador Confiança e Solidariedade $\left(\mathrm{X}_{2}\right)$, o desempenho de Porto dos Barcos chegou a 69\%. Os resultados apresentam que pouco se pode confiar na maioria das pessoas e somente algumas vezes as pessoas ajudam umas às outras.

Na COLUNA 4, constam as Escalas de Desempenho de cada indicador determinante de capital social na Comunidade de Oriente.

A Comunidade de Oriente obteve melhor desempenho no indicador Confiança e Solidariedade $\left(\mathrm{ED}_{22}=0,99\right)$, onde obteve um desempenho de $99 \%$. Isso significa que na comunidade existe um forte grau de confiança e solidariedade entre seus membros.

A Associação Comunitária dos Moradores de Oriente, que reúne todos os moradores de forma voluntária, em atividades como reuniões mensais, festas beneficentes, etc. A comunidade, formada por 79 famílias, que vieram da mesma árvore genealógica, mantém um bom relacionamento entre as famílias, tanto da própria comunidade como de comunidades vizinhas (Carvoeiro e Córrego de Volta), segundo o senhor Francisco Assis dos Santos (Assis Doce), presidente da Associação dos Moradores de Oriente.

A associação, representada pelas lideranças da comunidade, participa ativamente de reuniões na sede do município tanto na prefeitura local como sindicato e igreja. Ela realiza petições em busca de benefícios para a comunidade, além de um bom relacionamento com todas as secretarias municipais, o que facilita o trabalho em parceria na comunidade. A prova disso é o desempenho na variável Autoridade ou Capacitação e Ação Política ( $\left.\mathrm{X}_{6}\right)$, que foi de 83\%. Vale ressaltar, também, o trabalho da Associação na realização de dois assentamentos no Programa de Reforma Agrária Solidária (Paudarco e Cajueiro Encarnado); em dois subprojetos do Projeto São José I e II, de Eletrificação Rural e Abastecimento de Água, respectivamente, onde os moradores são beneficiados de forma direta ou indireta; e no PRONAF de infraestrutura, na construção de cacimbas na residência de cada morador da comunidade.

O indicador Coesão e Inclusão Social $\left(X_{5}\right.$ ) obteve um desempenho de 89,13\%. Na comunidade todos participam de projetos. A variável Informação e Comunidade $\left(\mathrm{X}_{4}\right)$ obteve o pior desempenho $\left(\mathrm{ED}_{24}=0,71\right.$, representando $\left.71 \%\right)$. Isso devido à distância de Oriente a sede do município $(32 \mathrm{~km})$, onde cerca de $3 \mathrm{~km}$ a estrada é de "piçarra” e nos meses de inverno, torna-se difícil o acesso. 
Na COLUNA 5, constam as contribuições de cada indicador na construção do Índice de Capital Social na Comunidade de Porto dos Barcos. A maior par cela foi do indicador Confiança e Solidariedade $\left(\mathrm{X}_{2}\right)$ com o valor de 0,16. Já a menor parcela foi do indicador Autoridade ou Capacitação e Ação Política $\left(\mathrm{X}_{6}\right)$ com o valor de 0,08. O Índice de Capital Social total na Comunidade de Porto dos Barcos foi de 0,67.

Na COLUNA 6, constam as contribuições de cada indicador na construção do Índice de Capital Social na Comunidade de Oriente. A maior parcela foi do indicador Confiança e Solidariedade $\left(\mathrm{X}_{2}\right)$, com o valor de 0,23. Enquanto que a menor contribuição foi do indicador Informação e Comunicação (X4) com o valor de 0,08. O Índice de Capital Social total na Comunidade de Oriente foi de 0,84 .

Na Relação do Índice Capital Social (RICS), a Comunidade de Oriente possui um Índice de Capital Social $(0,85)$, superior ao Índice Capital Social da Comunidade de Porto dos Barcos $(0,66)$ em 29\% (RICS = 1,29), devido ao acúmulo de compromissos da Comunidade de Oriente em benefício próprio, comprovado nos desempenhos de escala em 5 das 6 variáveis determinantes de capital social (grupos e redes, confiança e solidariedade, ação coletiva e cooperação, coesão e inclusão social e autoridade ou capacitação).

Na COLUNA 7, constam os valores da Relação da Escala de Desempenho de cada indicador entre as Comunidades de Oriente e Porto dos Barcos.

A Comunidade de Oriente obteve melhores desempenhos do que a Comunidade de Porto dos Barcos em 5 dos 6 indicadores. Somente no indicador Informação e Comunicação ( $\mathrm{X}_{4}$ ), a Comunidade de Porto dos Barcos obteve um desempenho superior a Oriente em $15 \%\left(\mathrm{RED}_{4}=0,85\right)$, decorrente da distância entre as duas comunidades em relação à sede do município, o que favorece a Comunidade de Porto dos Barcos a ter mais acesso à informação junto ao mercado local e serviços públicos.

No indicador Grupos e Redes ( $\mathrm{X}_{1}$ ), a Comunidade de Oriente obteve um desempenho superior à Comunidade de Porto dos Barcos em 39\% (RED $=1,39$ ). Isto significa que as associações de Oriente são mais atuantes, com presença de lideranças do que as associações de Porto dos Barcos, possuindo uma maior capacidade de gerar e acumular capital social; comprovada na contribuição do indicador, no qual o índice de Oriente foi de 0,16; enquanto que o índice de Porto dos Barcos foi de 0,11.

No indicador Confiança e Solidariedade $\left(\mathrm{X}_{2}\right)$, a Comunidade de Oriente obteve um desempenho melhor do que a Comunidade de Porto dos Barcos em 43\% $\left(\operatorname{RED}_{2}=1,43\right)$. Isto se deve a Comunidade de Oriente possuir maiores laços de confiança e solidariedade entre seus membros do que a Comunidade de Porto dos Barcos. 
Nos indicadores Ação Coletiva e Cooperação $\left(\mathrm{X}_{3}\right)$, Coesão e Inclusão Social $\left(\mathrm{X}_{5}\right)$ e Autoridade ou Capacitação e Ação Política $\left(\mathrm{X}_{6}\right)$, a Comunidade de Oriente também obteve desempenhos superiores a Comunidade de Porto dos Barcos em $25 \%, 25 \%$ e $19 \%$, respectivamente $\left(\mathrm{RED}_{3}=1,25 ; \mathrm{RED}_{5}=1,25 \mathrm{e} \mathrm{RED}_{6}=\right.$ 1,19). Esses resultados mostram que os moradores de Oriente se aplicam mais aos trabalhos coletivos, principalmente de forma voluntária, através de suas associações, do que os moradores de Porto dos Barcos.

\subsection{Análise do Índice de Qualidade de Vida nas Comuni- dades de Porto dos Barcos e Oriente}

Os resultados apresentados na Tabela 2 indicam as contribuições absolutas e relativas dos indicadores que compõem o índice de qualidade de vida nas Comunidades de Porto dos Barcos e Oriente.

Verificou-se que a maior contribuição para o Índice de Qualidade de Vida na Comunidade de Porto dos Barcos foi o indicador informação e comunicação $\left(\mathrm{I}_{1}\right)$, com a contribuição absoluta de 0,14 (representando uma contribuição relativa de 20\%), em decorrência da pequena distância entre a comunidade e a sede do município, o que facilita o acesso a informações gerais. Esse indicador foi maior, tanto em valores absolutos quanto relativos do que o mesmo indicador na Comunidade de Oriente; comprovado através da Relação entre os Índices de Qualidade de Vida (RIQV $)$, que apresentou um valor igual a 0,88 .

TABELA 2 - CONTRIBUIÇÃO DOS INDICADORES NA COMPOSIÇÃO DO ÍNDICE DE QUALIDADE DE VIDA NAS COMUNIDADES DE ORIENTE, PORTO DOS BARCOS, 2005

\begin{tabular}{l|c|cc|c|c}
\hline \multirow{2}{*}{ Indicadores } & \multicolumn{2}{|c|}{$\mathrm{IQV}_{2}(1)$} & \multicolumn{2}{c|}{$\mathrm{IQV}_{1}(2)$} & RIQV (3) \\
\cline { 2 - 7 } & & & & & \\
& VA & VR (\%) & VA & VR (\%) & VA \\
\hline Informação e Comunicação $\left(\mathrm{I}_{1}\right)$ & 0,13 & 15 & 0,14 & 20 & 0,93 \\
Conflito e Violência $\left(\mathrm{I}_{2}\right)$ & 0,17 & 19 & 0,13 & 18 & 1,31 \\
Saúde e Educação $\left(\mathrm{I}_{3}\right)$ & 0,14 & 16 & 0,12 & 17 & 1,17 \\
Habitação $\left(\mathrm{I}_{4}\right)$ & 0,17 & 19 & 0,13 & 18 & 1,31 \\
Condições Sanitárias e Higiene $\left(\mathrm{I}_{5}\right)$ & 0,17 & 19 & 0,13 & 18 & 1,31 \\
Econômico $\left(\mathrm{I}_{6}\right)$ & 0,11 & 12 & 0,06 & 8 & 1,83 \\
\hline Total & $\mathbf{0 , 8 9}$ & $\mathbf{1 0 0 , 0 0}$ & $\mathbf{0 , 7 1}$ & $\mathbf{1 0 0 , 0 0}$ & $\mathbf{1 , 2 5}$
\end{tabular}

Fonte: Dados da pesquisa.

1 - Índice de Qualidade de Vida na Comunidade de Oriente

2 - Índice de Qualidade de Vida na Comunidade de Porto dos Barcos

3 - Relação entre o Índice de Qualidade de Vida (RIQV) 
O indicador, com menor contribuição, foi o econômico ( $\left.\mathrm{I}_{6}\right)$, com contribuições absolutas e relativas iguais a 0,06 e 8\%, respectivamente. Valores esses muitos baixos, principalmente quando comparados com o indicador econômico de Oriente, que apresentou um resultado superior a $83 \%\left(\mathrm{RIQV}_{6}=1,83\right)$.

Os outros indicadores de Porto dos Barcos tiveram contribuições entre 17\% (indicador saúde e educação) a 18\% (indicadores conflito e violência, habitação e condições sanitárias e higiene). Mas quando comparamos com as contribuições dos mesmos indicadores em Oriente, nota-se que os indicadores possuem menores contribuições, tanto absolutas como relativas. No total, o Índice de Qualidade de Vida da Comunidade de Porto dos Barcos foi de 0,71.

Em relação ao Índice de Qualidade de Vida da Comunidade de Oriente, os indicadores de maiores contribuições foram: conflito e violência, habitação e condições sanitárias e higiene. Todos eles tiveram contribuições absolutas e relativas iguais a 0,17 e $19 \%$, respectivamente. O que comprova que Oriente é uma comunidade bastante pacífica, onde sua população reside em casas com boas infra estruturas, proporcionando um maior conforto, e tem cuidados com as condições sanitárias, o que possibilita um menor número de doenças.

A menor contribuição foi o indicador econômico, com contribuições absolutas e relativas iguais a $0,11 \mathrm{e} 12 \%$, respectivamente para a Comunidade de Oriente. Porém, quando comparado com os resultados de Porto dos Barcos (0,06 e $8 \%$ ), nota-se uma grande disparidade de resultado. Isso é comparado com Relação do Índice de Qualidade de Vida entre as duas comunidades, onde a contribuição de indicador de Oriente supera em $83 \%\left(\mathrm{RIQV}_{6}=1,83\right)$.

No total, o Índice de Qualidade de Vida de Oriente foi de 0,89 , superior ao Índice de Qualidade de Vida de Porto dos Barcos em 25\%.

\section{Conclusões e sugestões}

A primeira conclusão que se obtém a partir do presente trabalho, é a relevância do capital social como um fator preponderante da disparidade de desenvolvimento existente entre as comunidades de Oriente e Porto dos Barcos. A Comunidade de Oriente é mais desenvolvida, devido a seu maior estoque de capital social do que a Comunidade de Porto dos Barcos, a menos desenvolvida. Isso confirma a hipótese de que comunidades com maior estoque de capital social são mais desenvolvidas do que comunidades com menor estoque de capital social.

Apresentaram resultados favoráveis à Comunidade de Oriente em relação à Comunidade de Porto dos Barcos, 5 dos 6 indicadores determinantes de capital social. Apenas o indicador Informação - Comunicação apresentou resultado favorável a Comunidade de Porto dos Barcos. 
Dentre os indicadores determinantes de capital social na Comunidade de Oriente, destaca-se a variável Confiança e Solidariedade, que apresentou a maior contribuição para o Índice de Capital Social da comunidade. Isso confirma que comunidades mais solidárias e com forte grau de confiança mútua entre seus membros, terão maior capacidade de se desenvolver ou acumular capital social do que comunidades com baixo grau de confiança.

As associações existentes em Porto dos Barcos não são atuantes, como são as associações de Oriente, apresentando baixo grau de confiança entre seus integrantes, o que ocasiona no menor estoque de capital social do que Oriente. Isso não falseia que comunidade com maior grau de articulação social entre seus moradores tem maior capacidade de gerar e acumular capital social.

O maior estoque de capital social em Oriente tem produzido frutos, através de trabalhos realizados pelos moradores e lideranças das associações, reivindicando junto ao governo local, como por exemplo: dois subprojetos do Projeto São José I e II -PCPR ${ }^{10}$ (eletrificação rural e abastecimento de água, respectivamente), que beneficia toda comunidade de forma direta ou indireta; dois assentamentos do Programa de Reforma Agrária Solidária, que beneficia 16 famílias; e PRONAF de infraestrutura, para construção de cacimbas na residência de cada morador. Ou seja, é o acúmulo de capital social presente na Comunidade de Oriente, através da liderança, confiança, cooperação e articulação social que faz notório a eficácia institucional, proporcionando o desenvolvimento desta comunidade.

Os moradores de Oriente apresentam uma melhor qualidade de vida do que os moradores de Porto dos Barcos, devido a melhores disponibilidades de serviços relacionados à saúde e educação, com casas confortáveis, com melhores condições sanitárias e de higiene e maior renda média mensal do que os moradores de Porto dos Barcos.

As comunidades com maior estoque de capital social desfrutam de melhores índices de qualidade de vida.

Recomenda-se às autoridades, programar atividades que venham a alavancar a criação ou acúmulo de capital social nas comunidades; principalmente, neste caso, a Comunidade de Porto dos Barcos, que talvez seja um instrumento essencial para diminuir as disparidades de desenvolvimento local entre as comunidades, buscando assim melhorias na qualidade de vida de sua população.

${ }^{10}$ Programa de Combate à Pobreza Rural do Banco Mundial. 


\section{Referências bibliográficas}

ABRAHAM, A. \& PLATTEAU, J. P. (2001). Participatory Development in the Presence of Endogenous Community Imperfections. University of Namur, Belgium.

.(2004). 'Participatory Development: When Culture Creeps In'.In Culture and Public Action, V.Rao and M. Walton (editors), Stanford University Press.

ABU-EL-HAJ, J. (200o). A mobilização do capital social no Brasil: O caso da reforma sanitária no Ceará. São Paulo: Annablume.

BALLAND, J. M. \& PLATTEAU, J. P. (1999). 'The Ambiguous Impact of Inequality on Local Resource Management', World Development, 27(5): 773-88.

."Institutions and the Efficient Management of Environmental Resources", in Mähler, K.-G., and J.R. Vincent (eds). Handbook of Environmental Economics, Vol. 1,Amsterdam: Elsevier North-Holland, Chap. 4, pp. 127-90.

ABRAMOVAY, R. "A Formação do Capital Social para o Desenvolvimento Local Sustentável.” URL: http//www.capitalsocial.cjb.net (II Fórum contag de cooperação técnica. São Luís, 1998). 20p.

AMARAL FILHO, J. (2000). "Capital Social e desenvolvimento local no Ceará.” Jornal O Povo. Fortaleza, 26 de nov, p.o9.

AMARAL FILHO, J. (1995). "Desenvolvimento Regional Endógeno: (Re) construção de um conceito, reformulação das estratégias." Revista Econômica do Nordeste, Fortaleza,26(3):325-346.

BARRETO, R. C. S. \& KHAN, A. S. (2006). "Capital Social e Qualidade de Vida dos Assentamentos do Municípios de Caucaia-CE.” In: XLIV Congresso Brasileiro de Economia e Sociologia Rural, 2006, Fortaleza. Questões Agrárias, Educação no Campo e Desenvolvimento. Brasília : SOBER, v. 1. 2006. p. 1-20.

DURSTON, J. "Construyendo Capital Social Comunitario.” Revista de la CEPAL 69:103-118.

GOMEZ, J. A. (2000). "La calidad de vida y el tercer sector: nuevas dimensiones de la compeljidad.” URL: www.ceca.org.br/projeto/gvida.htm. Acesso em 14 de abril de 2000.

GROOTAERT, C. et al. (2003). "Questionário Integrado Para Medir Capital Social.” Banco Mundial, 73p.

HOLANDA, F. U. X. (2003). O Capital Social na Agricultura Familiar. Ações Cívicas tecendo o Desenvolvimento o caso do Assentamento Guriú. 2003. $279 f$. Tese (Doutorado em Sociologia). Programa de Pós-Graduação em Sociologia, Universidade Federal do Ceará, Fortaleza.

IPECE-Instituto de Pesquisa e Estratégica Econômica do Ceará (2004). "Perfil Básico Municipal: Itarema”. 10p.

IPLANCE-Instituto de Planejamento do Estado do Ceará (2001). "Índice de Desenvolvimento Municipal”. 103p.

KHAN, A. S. \& SILVA, L. M. R. "Capital Social das Comunidades Beneficiadas pelo Programa de Combate à Pobreza Rural -PCPR/Projeto São José -PSJ” - Estado do Ceará (2005). Revista de Economia e Sociologia Rural, Brasília-DF, v. 43, n. 1, p. 101-117. 
KHAN, A. S. (2002). “Reforma Agrária Solidária e Desenvolvimento Rural no Estado do Ceará.” In: KHAN, A. S. \& ROSA, A. L. T. (orgs.) Nordeste. Reflexões sobre Aspectos Setoriais e Locais de uma Economia. Fortaleza: CAEN, 328p.

.(2000). "Reforma Agrária Solidária e Extensão: novo modelo de desenvolvimento rural no Estado do Ceará.” UFC/CCA/DEA. Fortaleza, Ceará, 64p.

KLIKSBERG, Bernardo (1999).Capital Social y Claves Olvidadas del Desarrollo. INDES/BID, 32p.

MAYORGA, F. D. O \& KHAN, A. S. \& MAYORGA, R. D. \& LIMA, P. V. P. S.(2004). "Capital social, capital físico e a vulnerabilidade do sertanejo: o caso das comunidades de Lutsal e Sítio Lagoa no município de Tauá, Ceará”. Revista de Economia e Sociologia Rural, Rio de Janeiro, 42(1): 111-132, jan /mar 2004. 22p.

MEREGE, L. C. \& ALONSO, A. F. \& LÍCIO, E. (2000). “Desenvolvimento comunitário, metodologia e avaliação: o modelo da Associação Comunitária Monte Azul (ACOMA).” In: V Congresso Internacional Del CLAD sobre la Reforma y de la Administración Pública, Santo Domingo, Rep. Dominicana, 24-27 Oct. 2000. 20p.

MONASTÉRIO, Leonardo M. (1999). “Capital Social e Grupo de Interesse. Uma reflexão no Âmbito da Economia Regional.” URL: http//capitalsocial.cjb.net. (XXVII Encontro da Associação Nacional de Pós-Graduação em Economia. Belém-PA). 20p.

NILANDER, R. A. \& MATHIS, Armin (2001). “Índice de Qualidade de Vida Urbana de Barbacena” - IQVU: Experiência de Construção e Perspectivas de Aplicabilidade de um Instrumento Urbanístico para a Gestão Municipal. NAEA/UFPA. Belém, PA, 8p.

OSTROM, E. (1990). Governing the Commons: The Evolution of of Institutions for Collective Action, New York, Cambridge University Press.

PUTNAM, Robert D. (1996). Comunidade e Democracia: a Experiência da Itália Moderna. Rio de Janeiro: FGV, 364p.

TABOSA, F. J. S. \& MAYORGA, R. D. (2006) . “O Capital Social no Estado do Ceará: O Caso do Município de Itarema.” In: XLIV Congresso da Sociedade Brasileira de Economia e Sociologia Rural, 2006, Fortaleza-CE. Questões Agrárias, Educação no Campo e Desenvolvimento. Brasília-DF : SOBER, 2006. v. 1, p. 1-20.

TABOSA, F. J. S. \& TEIXEIRA, K. H. \& SILVA, D. M. F. \& MADALOZZO, C. L. \& Mayorga, M. I. O. (2004). "Desenvolvimento Local e Capital Social: uma Leitura sobre os núcleos e arranjos produtivos do Estado do Ceará”. In: XLII Congresso Brasileiro de Economia e Sociologia Rural, 2004. Anais. Brasília: SOBER. v. 1, p. 1-14.

WORLD BANK (2004). “Qué es capital social?” URL: www.worldbank.org/poverty/ spanish/scapital/scwhyrel2.htm . Acesso em 15 de julho de 2004.

Recebido em: 01 de outubro de 2008

Primeira resposta em: 05 de dezembro de 2008

Aceite em: 08 de janeiro de 2009 